Homology, Homotopy and Applications, vol.13(2), 2011, pp.197-215

\title{
SUPPORT VARIETIES AND REPRESENTATION TYPE OF SELF-INJECTIVE ALGEBRAS
}

\author{
JÖRG FELDVOSS AND SARAH WITHERSPOON
}

(communicated by Claude Cibils)

\begin{abstract}
We use the theory of varieties for modules arising from Hochschild cohomology to give an alternative version of the wildness criterion of Bergh and Solberg [7]: If a finite dimensional self-injective algebra has a module of complexity at least 3 and satisfies some finiteness assumptions on Hochschild cohomology, then the algebra is wild. We show directly how this is related to the analogous theory for Hopf algebras that we developed in [23]. We give applications to many different types of algebras: Hecke algebras, reduced universal enveloping algebras, small half-quantum groups, and Nichols (quantum symmetric) algebras.
\end{abstract}

\section{Introduction}

The cohomology of a finite dimensional algebra holds information about its representation type. Rickard first made this observation about group algebras, and it inspired him to develop a theory for self-injective algebras (see [41]). Since then mathematicians have applied homological methods to determine representation type in various contexts (see $[\mathbf{2}, \mathbf{3}, \mathbf{2 0}, \mathbf{3 0}, \mathbf{3 1}, \mathbf{4 0}]$ ), and they have partially filled a gap in a wildness criterion of Rickard [41] (see [2, Corrigendum] and [7, 22, 23]).

For example, Farnsteiner [22] proved that if the Krull dimension of the cohomology ring of a cocommutative Hopf algebra is at least 3, then the Hopf algebra has wild representation type; more precisely he gave a block version of this statement. He used the result of Friedlander and Suslin $[\mathbf{2 6}]$ that the cohomology ring of a finite dimensional cocommutative Hopf algebra is finitely generated, as well as the theory of support varieties developed by Suslin, Friedlander, Bendel, and Pevtsova $[\mathbf{2 5}, \mathbf{4 5}]$. In $[\mathbf{2 3}]$ we gave a direct generalization of Farnsteiner's results to all Hopf algebras satisfying finiteness assumptions on their cohomology. Bergh and Solberg [7] gave a

The second author was partially supported by NSF grant \#DMS-0800832 and Advanced Research Program Grant 010366-0046-2007 from the Texas Higher Education Coordinating Board.

Received May 12, 2011, revised September 8, 2011; published on November 25, 2011.

2010 Mathematics Subject Classification: 16D50, 16L60, 16G60, 16G10, 16E40, 16T05, 17B35, 17B37, $20 \mathrm{C} 08$.

Key words and phrases: support variety, Hochschild cohomology, complexity, representation type, wild, tame, block, self-injective algebra, Hecke algebra, reduced universal enveloping algebra, small half-quantum group, Nichols algebra, quantum symmetric algebra, Hopf algebra.

Article available at http://intlpress.com/HHA/v13/n2/a13 and doi:10.4310/HHA.2011.v13.n2.a13

Copyright (C) 2011, International Press. Permission to copy for private use granted. 
wildness criterion even more generally for self-injective algebras, under some finiteness assumptions on the cohomology of given modules.

In this paper, we begin by showing how a wildness criterion for self-injective algebras may be obtained using Hochschild cohomology and the theory of support varieties developed for Hochschild cohomology by Erdmann, Holloway, Snashall, Solberg, and Taillefer (see [19] and [42]). Bergh and Solberg have weaker hypotheses than ours (see, for example, [7, Example 4.5]) due to the flexibility their relative theory provides, however our finiteness assumptions are known to hold for many types of algebras. Most of this article is devoted to applications: We apply the theory to Hecke algebras, reduced universal enveloping algebras of restricted Lie algebras, small halfquantum groups, and Nichols (quantum symmetric) algebras. In the last section, we show that for a Hopf algebra, one may make a choice so that the support varieties for a module defined in terms of Hochschild cohomology herein are isomorphic to the support varieties defined in [23] in terms of the cohomology ring of the Hopf algebra. Thus we give a direct connection between these two seemingly different support variety theories for Hopf algebras.

Throughout this paper we will assume that all associative rings have unity elements and that all modules over associative rings are unital. Moreover, all modules are left modules and all tensor products are over the ground field unless indicated otherwise. Finally, the principal block of an augmented $k$-algebra $A$ is the unique indecomposable ideal direct summand of $A$ whose unity element acts as the identity on the trivial $A$-module $k$.

\section{Complexity and support varieties}

Let $A$ be a finite dimensional self-injective algebra over an algebraically closed field $k$ of arbitrary characteristic unless stated otherwise. In this section we recall some definitions and results that will be needed later.

Let $A^{e}:=A \otimes A^{\mathrm{op}}$ denote the enveloping algebra of $A$. Then the graded ring $\operatorname{Ext}_{A^{e}}(A, A)$ is isomorphic to the Hochschild cohomology ring $\mathrm{HH}^{*}(A)$ of $A$. For any $A$-bimodule $M, \operatorname{Ext}_{A^{e}}(A, M)$ is isomorphic to the Hochschild cohomology $\mathrm{HH}^{*}(A, M)$.

We make the following assumption in order to obtain an affine variety, namely $\mathcal{V}_{H}$ defined below, in which to consider the support varieties of finite dimensional $A$-modules.

Assumption (fg1). There is a graded subalgebra $H^{*}$ of $\mathrm{HH}^{*}(A)$ such that $H^{*}$ is a finitely generated commutative algebra and $H^{0}=\mathrm{HH}^{0}(A)$.

For any $A$-module $M$ there is a homomorphism of graded rings $\varphi_{M}: \mathrm{HH}^{\bullet}(A) \rightarrow$ $\operatorname{Ext}_{A}(M, M)$ given by $\varphi_{M}(\eta):=\eta \otimes_{A} M$ (see [42, p. 707]). Combined with Yoneda composition, the homomorphisms $\varphi_{M}$ and $\varphi_{N}$ induce right and left $\mathrm{HH}^{*}(A)$-actions on $\operatorname{Ext}_{A}{ }_{A}(M, N)$ for any two $A$-modules $M$ and $N$ (see [42, p. 707]), and by restriction they also induce right and left $H^{*}$-actions on $\operatorname{Ext}_{A}{ }^{\circ}(M, N)$.

Denote by $\mathcal{N}_{H}$ the ideal in $H^{\bullet}$ generated by the homogeneous nilpotent elements of $H^{\bullet}$ and the Jacobson radical of $H^{0}$. Since $\mathcal{N}_{H}$ is a homogeneous ideal of $H^{\bullet}$ and the latter is commutative by assumption (fg1), it follows from [42, Lemma 2.2(b)] that every maximal ideal of $H^{*}$ contains $\mathcal{N}_{H}$. Consequently, there is a bijection between 
the maximal ideals of $H^{\bullet}$ and the maximal ideals of $\overline{H^{\bullet}}:=H^{\bullet} / \mathcal{N}_{H}$. In the following we denote by $\mathcal{V}_{H}$ the maximal ideal spectrum of $\overline{H^{\circ}}$.

Now let $M$ and $N$ be finite dimensional $A$-modules. Let $I_{H}(M, N)$ be the subset of those elements in $\mathcal{V}_{H}$ whose inverse images in $H^{\bullet}$ contain the annihilator of the action of $H^{*}$ on $\operatorname{Ext}_{A}{ }^{*}(M, N)$. (The action may be taken to be either the left or right action; their annihilators coincide according to [42, Lemma 2.1].) Then $I_{H}(M, N)$ is a homogeneous ideal of $\overline{H^{\circ}}$. Let $\mathcal{V}_{H}(M, N)$ denote the maximal ideal spectrum of the finitely generated commutative $k$-algebra $\overline{H^{*}} / I_{H}(M, N)$. As the ideal $I_{H}(M, N)$ is homogeneous, the variety $\mathcal{V}_{H}(M, N)$ is conical. If $M=N$, we write $I_{H}(M):=$ $I_{H}(M, M)$ and $\mathcal{V}_{H}(M):=\mathcal{V}_{H}(M, M)$. The latter is called the support variety of $M$ (see [42, Definition 2.3]).

In order to be able to apply the results of $[\mathbf{1 9}]$ and $[\mathbf{4 2}]$, we also need the following assumption, where $\operatorname{Jac}(A)$ denotes the Jacobson radical of $A$.

Assumption (fg2). $\operatorname{Ext}_{A}{ }^{\prime}(A / \operatorname{Jac}(A), A / \operatorname{Jac}(A))$ is a finitely generated $H^{*}$-module.

Remark 1.1. By [19, Proposition 2.4], the assumption (fg2) is equivalent to either of the following two statements:

(i) For all finite dimensional $A$-modules $M$ and $N, \operatorname{Ext}_{A}^{\circ}(M, N)$ is finitely generated over $H^{*}$.

(ii) For all finite dimensional $A$-bimodules $M, \operatorname{HH}^{*}(A, M)$ is finitely generated over $H^{\circ}$.

Further, the statement (i) is equivalent to the corresponding statement in which $M=N$, since we may apply the latter to the direct sum of two finite dimensional modules to obtain (i).

In the following we show that every block of a finite dimensional self-injective algebra for which assumptions (fg1) and (fg2) hold is self-injective and also satisfies the same conditions (fg1) and (fg2).

Lemma 1.2. Let $A$ be a finite dimensional algebra over an arbitrary field $k$ and let $B$ be a block of $A$. Then the following statements hold:

1. If $A$ is self-injective, then $B$ is self-injective.

2. If A satisfies (fg1), then B satisfies (fg1).

3. If A satisfies (fg2), then $B$ satisfies (fg2).

Proof. (1) Since direct summands of injective modules are injective, $B$ is injective as an $A$-module and therefore also as a $B$-module.

(2) Let $e$ be the primitive central idempotent of $A$ corresponding to $B$, and consider the algebra homomorphism from $A^{e}$ onto $B^{e}$ defined by $a \otimes a^{\prime} \mapsto e a \otimes e a^{\prime}$. Letting $e$ act on $f \in \operatorname{Hom}_{k}\left(A^{\otimes n}, A\right)$ as $(e f)\left(x_{1} \otimes \cdots \otimes x_{n}\right)=e\left(f\left(e x_{1} \otimes \cdots \otimes e x_{n}\right)\right)$, one sees that $\mathrm{HH}^{n}(B)$ is isomorphic to $e \mathrm{HH}^{n}(A)$ where the latter is a direct summand of $\mathrm{HH}^{n}(A)$ for every non-negative integer $n$. In particular, $e H^{\bullet}$ is a finitely generated commutative subalgebra of the direct summand $\mathrm{HH}^{*}(B)=e \mathrm{HH}^{*}(A)$ of $\mathrm{HH}^{*}(A)$. Hence $B$ satisfies (fg1).

(3) For all $A$-modules $M$ and $N, \operatorname{Ext}_{B}^{n}(e M, e N)$ is isomorphic to $e \operatorname{Ext}_{A}^{n}(M, N)$ where the latter is a direct summand of $\operatorname{Ext}_{A}^{n}(M, N)$. If $\xi_{1}, \ldots, \xi_{s}$ are generators 
for the $H^{\bullet}$-module $\operatorname{Ext}_{A}^{\bullet}(M, N)$, then $e \xi_{1}, \ldots, e \xi_{s}$ are generators for the $e H^{\bullet}$-module $\operatorname{Ext}_{B}(e M, e N)$. Hence B satisfies (fg2).

Let $V$. be a graded vector space over $k$ with finite dimensional homogeneous components. The rate of growth $\gamma\left(V_{\bullet}\right)$ of $V_{\bullet}$ is the smallest non-negative integer $c$ such that there is a number $b$ for which $\operatorname{dim}_{k} V_{n} \leqslant b n^{c-1}$ for all positive integers $n$. If no such $c$ exists, then $\gamma\left(V_{\bullet}\right)$ is defined to be $\infty$. The complexity $\operatorname{cx}_{A}(M)$ of an $A$-module $M$ may be defined in the standard way: Let $P_{\bullet}: \cdots \rightarrow P_{1} \rightarrow P_{0} \rightarrow M \rightarrow 0$ be a minimal projective resolution of $M$. Then $\operatorname{cx}_{A}(M):=\gamma(P \bullet)$.

We will need the following lemma stating that for a module belonging to a block of a finite dimensional self-injective algebra, the complexity is the same whether it is considered as a module for the block or for the whole algebra.

Lemma 1.3. Let $A$ be a finite dimensional self-injective algebra over an arbitrary field and let $B$ be a block of $A$. Then $\operatorname{cx}_{B}(M)=\operatorname{cx}_{A}(M)$ for every $B$-module $M$.

Proof. Every projective $A$-module is projective as a $B$-module, and consequently $\operatorname{cx}_{B}(M) \leqslant \operatorname{cx}_{A}(M)$. On the other hand, $B$ is a direct summand of $A$, and as such $B$ is a projective $A$-module. Hence every projective $B$-module is also projective as an $A$-module and therefore $\operatorname{cx}_{B}(M) \geqslant \operatorname{cx}_{A}(M)$.

Finally, we will need the following connection between complexity, support varieties, and the rate of growth of the self-extensions of a module. The first equality is just [19, Theorem 2.5(c)]. Note that the indecomposability of $A$ is not used in the proof (see the proof of $[\mathbf{1 9}$, Proposition 2.1(a)]). The second equality is implicitly contained in the suggested proof (see also the first part of the proof of [23, Proposition 2.3] for Hopf algebras that transfers to this more general situation under the assumptions (fg1) and (fg2).

Theorem 1.4. Let $A$ be a finite dimensional self-injective algebra over an algebraically closed field $k$ of arbitrary characteristic for which assumptions (fg1) and (fg2) hold. Let $M$ be a finite dimensional A-module. Then

$$
\operatorname{cx}_{A}(M)=\operatorname{dim} \mathcal{V}_{H}(M)=\gamma\left(\operatorname{Ext}_{A}(M, M)\right)
$$

\section{Complexity and representation type}

Theorem 2.1 below generalizes [22, Theorem 3.1] and [23, Theorem 3.1] (cf. [7, Theorem 4.1]). Thus we recover a result of Rickard [41, Theorem 2], whose proof contains a gap (see [2, Corrigendum] and [22]), under the assumptions (fg1) and (fg2). We apply the general theory developed in [19] and [42] (the needed parts of which are summarized in Section 1) to adapt Farnsteiner's proof to this more general setting. Theorem 2.1 is similar to [7, Theorem 4.1] with slightly different hypotheses; for completeness we give a proof in our context.

Recall that there are three classes of finite dimensional associative algebras over an algebraically closed field (see [12, Corollary C] or [5, Theorem 4.4.2]): An algebra $A$ is representation-finite if there are only finitely many isomorphism classes of finite dimensional indecomposable $A$-modules. It is tame if it is not representation-finite and if the isomorphism classes of indecomposable $A$-modules in any fixed dimension 
are almost all contained in a finite number of 1-parameter families. The algebra $A$ is wild if the category of finite dimensional $A$-modules contains the category of finite dimensional modules over the free associative algebra in two indeterminates. (For more precise definitions we refer the reader to [5, Definition 4.4.1].) The classification of indecomposable objects (up to isomorphism) of the latter category is a well-known unsolvable problem, and so one is only able to classify the finite dimensional indecomposable modules of representation-finite or tame algebras.

Theorem 2.1. Let $A$ be a finite dimensional self-injective algebra over an algebraically closed field $k$ of arbitrary characteristic for which assumptions (fg1) and (fg2) hold and let $B$ be a block of $A$. If there is a B-module $M$ such that $\operatorname{cx}_{A}(M) \geqslant 3$, then $B$ is wild.

Proof. Let $M$ be a $B$-module for which $\operatorname{cx}_{A}(M) \geqslant 3$. It follows from Lemma 1.3 that $\mathrm{Cx}_{B}(M)=\mathrm{cx}_{A}(M) \geqslant 3$. According to Lemma 1.2, $B$ is also a finite dimensional selfinjective algebra over $k$ for which assumptions (fg1) and (fg2) hold. As a consequence, it is enough to prove the assertion for $A$ and one can assume that $A=B$.

It follows from Theorem 1.4 and our hypotheses that $n:=\operatorname{dim} \mathcal{V}_{H}(M)=\operatorname{cx}_{A}(M)$ $\geqslant 3$. For each $\zeta \in \overline{H^{*}}$, denote by $\langle\zeta\rangle$ the ideal of $\overline{H^{*}}$ generated by $\zeta$. For each ideal $I$ of $\overline{H^{*}}$, denote by $Z(I)$ its zero set, that is the set of all maximal ideals of $\overline{H^{*}}$ containing $I$. Since $\mathcal{V}_{H}(M)$ is a conical subvariety of $\mathcal{V}_{H}$, an application of the Noether Normalization Lemma (see [22, Lemma 1.1]) yields non-zero elements $\eta_{s}(s \in k)$ in $\overline{H^{d}}$ for some $d>0$ such that

(i) $\operatorname{dim} Z\left(\left\langle\eta_{s}\right\rangle\right) \cap \mathcal{V}_{H}(M)=n-1$ for all $s \in k$,

(ii) $\operatorname{dim} Z\left(\left\langle\eta_{s}\right\rangle\right) \cap Z\left(\left\langle\eta_{t}\right\rangle\right) \cap \mathcal{V}_{H}(M)=n-2$ for $s \neq t \in k$.

According to [19, Proposition 4.3 and Definition 3.1], there are $A$-bimodules $M_{\eta_{s}}$ for which the corresponding $A$-modules $N_{\eta_{s}}:=M_{\eta_{s}} \otimes_{A} M$ satisfy

$$
\mathcal{V}_{H}\left(N_{\eta_{s}}\right)=Z\left(\left\langle\eta_{s}\right\rangle\right) \cap \mathcal{V}_{H}(M) \text { and } \operatorname{dim}_{k} N_{\eta_{s}} \leqslant b_{A},
$$

where $b_{A}:=\left(\operatorname{dim}_{k} M\right)\left(\operatorname{dim}_{k} A\right)\left(\operatorname{dim}_{k} \Omega_{A^{e}}^{d-1}(A)\right)$, for all $s \in k$. Decompose $N_{\eta_{s}}$ into a direct sum of indecomposable $A$-modules. By [42, Proposition 3.4(f)] and (i) above, for each $s$ there is an indecomposable direct summand $X_{s}$ of $N_{\eta_{s}}$ such that

$$
\mathcal{V}_{H}\left(X_{s}\right) \subseteq \mathcal{V}_{H}\left(N_{\eta_{s}}\right)=Z\left(\left\langle\eta_{s}\right\rangle\right) \cap \mathcal{V}_{H}(M)
$$

and $\operatorname{dim} \mathcal{V}_{H}\left(X_{s}\right)=n-1$. We claim that $\mathcal{V}_{H}\left(X_{s}\right) \neq \mathcal{V}_{H}\left(X_{t}\right)$ when $s \neq t$ : Note that if $\mathcal{V}_{H}\left(X_{s}\right)=\mathcal{V}_{H}\left(X_{t}\right)$, then

$$
\mathcal{V}_{H}\left(X_{s}\right) \subseteq Z\left(\left\langle\eta_{s}\right\rangle\right) \cap Z\left(\left\langle\eta_{t}\right\rangle\right) \cap \mathcal{V}_{H}(M)
$$

and the latter variety has dimension $n-2$ if $s \neq t$. It follows that the varieties $\mathcal{V}_{H}\left(X_{s}\right)$ are distinct for different values of $s$, implying that the indecomposable $A$-modules $X_{s}$ are pairwise non-isomorphic. The dimensions of the modules $X_{s}$ are all bounded by $b_{A}$, since $X_{s}$ is a direct summand of $N_{\eta_{s}}$. Consequently, there are infinitely many non-isomorphic indecomposable $A$-modules $X_{s}$ of some fixed dimension. According to Theorem 1.4, $\operatorname{cx}_{A}\left(X_{s}\right)=\operatorname{dim} \mathcal{V}_{H}\left(X_{s}\right)=n-1 \geqslant 2$.

If $A$ is not wild, then by [12, Corollary $\mathrm{C}], A$ is tame or representation-finite. It follows from $[\mathbf{1 2}$, Theorem $\mathrm{D}]$ that only finitely many indecomposable $A$-modules of any dimension (up to isomorphism) are not isomorphic to their Auslander-Reiten 
translates. Since $A$ is self-injective, the Auslander-Reiten translation $\tau_{A}$ is the same as $\mathcal{N}_{A} \circ \Omega_{A}^{2}=\Omega_{A}^{2} \circ \mathcal{N}_{A}$ where $\mathcal{N}_{A}$ denotes the Nakayama functor of $A$ (see [4, Proposition IV.3.7(a)]). The Nakayama functor preserves projective modules and so any $A$-module isomorphic to its Auslander-Reiten translate is periodic and thus has complexity 1 . Hence in any dimension there are only finitely many isomorphism classes of indecomposable $A$-modules with complexity not equal to 1 . This is a contradiction, since we have shown that for some dimension, there are infinitely many nonisomorphic indecomposable $A$-modules of complexity greater than 1 . Therefore $A$ is wild.

An application of Theorem 2.1 and the Trichotomy Theorem [12, Corollary C] is

Corollary 2.2. Let $A$ be a finite dimensional self-injective algebra over an algebraically closed field $k$ of arbitrary characteristic for which assumptions (fg1) and (fg2) hold and let $B$ be a block of $A$. If $B$ is tame, then $\operatorname{cx}_{A}(M) \leqslant 2$ for every finite dimensional $B$-module $M$.

Remark 2.3. Note that the tameness of an algebra $A$ implies the tameness of every block of $A$. In particular, Corollary 2.2 holds also for the whole algebra.

\section{Hecke algebras}

Let $\mathcal{H}_{q}$ be the Hecke algebra associated to a finite Coxeter group of classical type over a field $k$, with non-zero parameter $q$ in $k$. It is well-known that $\mathcal{H}_{q}$ is self-injective (see [15, Theorem 2.3] for type A and [15, Remark after Theorem 2.8] for types B and D). Let $H^{\bullet}:=\operatorname{HH}^{\text {ev }}\left(\mathcal{H}_{q}\right):=\bigoplus_{n \geqslant 0} \operatorname{HH}^{2 n}\left(\mathcal{H}_{q}\right)$, the subalgebra of $\mathrm{HH}^{\circ}\left(\mathcal{H}_{q}\right)$ generated by elements of even degree. It follows from a recent result of Linckelmann [34, Theorem 1.1], Remark 1.1, and [27, Corollary 1, p. 281] that assumptions (fg1) and (fg2) hold for $\mathcal{H}_{q}$ as long as the characteristic of the ground field is zero and the order of $q$ is odd if $\mathcal{H}_{q}$ is of type $\mathrm{B}$ or $\mathrm{D}$, as stated in the next theorem. To obtain this statement for the even part of the Hochschild cohomology from Linckelmann's result, first note that we may take as generators of the full Hochschild cohomology ring a finite set of homogeneous elements. If we take those generators of even degree combined with all products of pairs of generators in odd degree, we obtain a finite set of generators of the even subring, since squares of odd degree elements are zero. A similar argument applies to modules over the Hochschild cohomology ring.

Theorem 3.1. Assume that the characteristic of $k$ is zero. Let $q$ be a primitive $\ell$ th root of unity in $k$, for some integer $\ell>1$, and assume that $\ell$ is an odd integer if $\mathcal{H}_{q}$ is of type $\mathrm{B}$ or $\mathrm{D}$. Then the even Hochschild cohomology ring $\mathrm{HH}^{\mathrm{ev}}\left(\mathcal{H}_{q}\right)$ is a finitely generated commutative algebra. Moreover, $\operatorname{Ext}_{\mathcal{H}_{q}}(M, N)$ is finitely generated as an $\mathrm{HH}^{\mathrm{ev}}\left(\mathcal{H}_{q}\right)$-module for all finite dimensional $\mathcal{H}_{q}$-modules $M$ and $N$.

A crucial tool in finding the representation types of blocks of Hecke algebras by applying Theorem 2.1 is the following lemma.

Lemma 3.2. If $A$ and $A^{\prime}$ are finite dimensional self-injective augmented algebras over an arbitrary field $k$ for which assumptions (fg1) and (fg2) hold, then $\mathrm{cx}_{A \otimes A^{\prime}}(k)=$ $\mathrm{cx}_{A}(k)+\mathrm{cx}_{A^{\prime}}(k)$. 
Proof. By the Künneth Theorem, the tensor product of projective resolutions of $k$ as an $A$-module and as an $A^{\prime}$-module is a projective resolution of $k$ as an $A \otimes A^{\prime}$-module, and moreover, $\operatorname{Ext}_{A \otimes A^{\prime}}(k, k) \cong \operatorname{Ext}_{A}{ }_{A}(k, k) \otimes \operatorname{Ext}_{A^{\prime}}(k, k)$. It is well-known that the Hochschild cohomology of a tensor product of algebras is the super tensor product of the Hochschild cohomology of the factors (see, e.g., [35, Theorem 4.2.5] for the analog for Hochschild homology). As (fg1) and (fg2) hold for $A$ and $A^{\prime}$ by hypothesis, (fg1) and (fg2) also hold for $A \otimes A^{\prime}$. Hence the result follows from Theorem 1.4. (Note that the proof of Theorem 1.4 does not use the self-injectivity of the algebra.)

Let $\mathcal{H}_{q}\left(\mathrm{~A}_{r}\right)$ denote the Hecke algebra of type $\mathrm{A}_{r}$ at a primitive root of unity $q$ of order $\ell$ where $2 \leqslant \ell \leqslant r$. Erdmann and Nakano [20] investigated the representation types of the blocks of $\mathcal{H}_{q}\left(\mathrm{~A}_{r}\right)$ over an arbitrary field. (Note that according to $[\mathbf{1 6}$, Theorem 4.3], $\mathcal{H}_{q}\left(\mathrm{~A}_{r}\right)$ is semisimple unless $2 \leqslant \ell \leqslant r$. $)$ The simple $\mathcal{H}_{q}\left(\mathrm{~A}_{r}\right)$-modules are in bijection with the $\ell$-regular partitions of $r$ (see [15, Theorem 7.6]). Let $D^{\lambda}$ denote the simple $\mathcal{H}_{q}\left(\mathrm{~A}_{r}\right)$-module corresponding to the $\ell$-regular partition $\lambda$. Then $D^{\lambda}$ and $D^{\mu}$ belong to the same block of $\mathcal{H}_{q}\left(\mathrm{~A}_{r}\right)$ if and only if $\lambda$ and $\mu$ have the same $\ell$-core (see [16, Theorem 4.13]). The $\ell$-core of a partition $\lambda$ of $r$ is the partition whose Young diagram is obtained from the Young diagram of $\lambda$ by removing as many rim $\ell$-hooks as possible. Let $\mathcal{B}_{\lambda}$ denote the block of $\mathcal{H}_{q}\left(\mathrm{~A}_{r}\right)$ that contains the simple module $D^{\lambda}$. The weight $w(\lambda)$ of the block $\mathcal{B}_{\lambda}$ is defined by $|\gamma|+\ell \cdot w(\lambda)=r$, where $\gamma$ is the $\ell$-core of $\lambda$.

Erdmann and Nakano [20, Proposition 3.3(A)] gave a proof of the following result for fields of arbitrary characteristic by applying Rickard's wildness criterion [41, Theorem 2] whose proof contains a gap. By using our Theorem 2.1 and Linckelmann's result (Theorem 3.1) we can recover the result of Erdmann and Nakano for fields of characteristic zero.

Theorem 3.3. Let $\mathcal{B}_{\lambda}$ be a block of the Hecke algebra $\mathcal{H}_{q}\left(\mathrm{~A}_{r}\right)$ over an algebraically closed field $k$ of characteristic zero at a primitive root of unity $q$ in $k$ of order $\ell$, where $2 \leqslant \ell \leqslant r$. If $w(\lambda) \geqslant 3$, then $\mathcal{B}_{\lambda}$ is wild.

Proof. Let $Y^{\lambda}$ denote the $q$-Young module that is the unique indecomposable direct summand of the $q$-permutation module of the partition $\lambda$ containing $D^{\lambda}$ (see $[\mathbf{1 4}$, Section 2]). Let $\mathcal{H}_{q}(\rho)$ denote the $q$-Young vertex of $Y^{\lambda}$. Erdmann and Nakano [20, Theorem 2.2] proved that $\operatorname{cx}_{\mathcal{H}_{q}\left(\mathrm{~A}_{r}\right)}\left(Y^{\lambda}\right)=\operatorname{cx}_{\mathcal{H}_{q}(\rho)}(k)$. As $w(\lambda) \geqslant 3, \mathcal{H}_{q}(\rho)$ is a free module over the subalgebra $\mathcal{H}_{q}\left(\mathrm{~A}_{\ell}\right) \otimes \mathcal{H}_{q}\left(\mathrm{~A}_{\ell}\right) \otimes \mathcal{H}_{q}\left(\mathrm{~A}_{\ell}\right)$ (see [20, proof of Proposition $3.3(\mathrm{~A})])$. By virtue of [16, Theorem 4.3], $\mathcal{H}_{q}\left(\mathrm{~A}_{\ell}\right)$ is not semisimple and therefore $\operatorname{cx}_{\mathcal{H}_{q}\left(\mathrm{~A}_{\ell}\right)}(k) \geqslant 1$. It follows from the above and Lemma 3.2 that

$$
\operatorname{cx}_{\mathcal{H}_{q}\left(\mathrm{~A}_{r}\right)}\left(Y^{\lambda}\right)=\operatorname{cx}_{\mathcal{H}_{q}(\rho)}(k) \geqslant \operatorname{cx}_{\mathcal{H}_{q}\left(\mathrm{~A}_{\ell}\right) \otimes \mathcal{H}_{q}\left(\mathrm{~A}_{\ell}\right) \otimes \mathcal{H}_{q}\left(\mathrm{~A}_{\ell}\right)}(k)=3 \cdot \operatorname{cx}_{\mathcal{H}_{q}\left(\mathrm{~A}_{\ell}\right)}(k) \geqslant 3 .
$$

Now the $q$-Young module $Y^{\lambda}$ belongs to $\mathcal{B}_{\lambda}$ by definition, and thus Theorem 2.1 in conjunction with Theorem 3.1 yields the assertion.

Remark 3.4. For the principal block of the Hecke algebra $\mathcal{H}_{q}\left(\mathrm{~A}_{r}\right)$, the conclusion of Theorem 3.3 is also an immediate consequence of Theorem 1.4, [6, Theorem 1.1], and Theorem 2.1.

Linckelmann's result [34, Theorem 1.1] neither covers the two-parameter Hecke algebras nor the one-parameter Hecke algebras of types B and D at parameters of even 
order. Ariki applies Rickard's wildness criterion in his paper only in the two-parameter case or for parameters of degree two (see [2, Corrigendum]), so Theorem 2.1 cannot be used to give an alternative approach to fix the gap as for type A. On the other hand, as in Remark 3.4, we obtain from Theorem 1.4 and [6, Theorem 6.2, Theorem 6.6, and Theorem 1.1] in conjunction with Theorem 2.1 the following result.

Theorem 3.5. Let $\mathcal{H}_{q}$ be the Hecke algebra of type $\mathrm{B}_{r}$ or $\mathrm{D}_{r}$ over an algebraically closed field $k$ of characteristic zero at a primitive $\ell$ th root $q$ of unity in $k$ for some odd integer $\ell>1$. If $r \geqslant 3 \ell$, then the principal block of $\mathcal{H}_{q}$ is wild.

Now let $\mathcal{H}_{q}$ be the Hecke algebra of type $\mathrm{B}_{r}$ or $\mathrm{D}_{r}$ over an arbitrary field $k$ of characteristic zero at a primitive $\ell$ th root $q$ of unity in $k$ for some odd integer $\ell>1$. Then it follows from [17, Theorem 4.17] (see also [2, Theorem 38]) for type $\mathrm{B}_{r},[\mathbf{3 9}$, (3.6) and (3.7)] for type $\mathrm{D}_{r}$ ( $r$ odd), and [32, Main result, p. 410] for type $\mathrm{D}_{r}$ ( $r$ even) in conjunction with Uno's result for type A (see $[\mathbf{3},(1.1)]$ ) that $\mathcal{H}_{q}$ is representationfinite if and only if $r<2 \ell$ (see also [3, pp. 135-136] for the two-parameter Hecke algebra). Consequently, Theorem 3.5 reduces the proof of [2, Theorem 57(1)] to the cases $2 \ell \leqslant r<3 \ell$ in which, according to Linckelmann's result [34, Theorem 1.2] and Theorem 1.4, all $\mathcal{H}_{q}$-modules have complexity at most 2 and therefore a wildness criterion à la Rickard would never apply.

On the other hand, a similar argument as above can be used to give a short proof for the wildness of the Hecke algebra $\mathcal{H}_{q}$ of type $\mathrm{B}_{r}$ or $\mathrm{D}_{r}$ over an arbitrary field $k$ of characteristic $\neq 2$ at a primitive $\ell$ th root $q$ of unity in $k$ for some odd integer $\ell>1$ by applying [20, Proposition 3.3(B)]. According to the latter, $\mathcal{H}_{q}\left(\mathrm{~A}_{2 \ell}\right)$ is wild. Then [17, Theorem 4.17] (or [2, Theorem 38]) for type B and [32, Main result, p. 410] for type $\mathrm{D}$ imply that $\mathcal{H}_{q}\left(\mathrm{~B}_{2 \ell}\right)$ and $\mathcal{H}_{q}\left(\mathrm{D}_{2 \ell}\right)$ are wild as they contain $\mathcal{H}_{q}\left(\mathrm{~A}_{2 \ell}\right)$ as an ideal direct summand. Now [2, Corollary $4(2)]$ shows that $\mathcal{H}_{q}\left(\mathrm{~B}_{r}\right)$ and $\mathcal{H}_{q}\left(\mathrm{D}_{r}\right)$ are wild as long as $n \geqslant 2 \ell$.

\section{Reduced universal enveloping algebras}

Let $\mathfrak{g}$ be a finite dimensional restricted Lie algebra over a field $k$ of prime characteristic, let $\chi$ be any linear form on $\mathfrak{g}$, and let $u(\mathfrak{g}, \chi)$ denote the $\chi$-reduced universal enveloping algebra of $\mathfrak{g}$ (see [44, Section 5.3]). Note that $u(\mathfrak{g}, \chi)$ is always a Frobenius algebra (see [44, Corollary 5.4.3]), and therefore $u(\mathfrak{g}, \chi)$ is self-injective but it is a Hopf algebra only if $\chi=0$. In this section we give a unified proof of the wildness criterion for $\chi$-reduced universal enveloping algebras. In [22, Theorem 4.1 and Corollary 4.2] this was done by repeating parts of the proof for $\chi=0$ in the general case. We need the following result assuring that assumptions (fg1) and (fg2) hold for finite dimensional $\chi$-reduced universal enveloping algebras.

Lemma 4.1. Let $\mathfrak{g}$ be a finite dimensional restricted Lie algebra over a field $k$ of prime characteristic and let $\chi$ be any linear form on $\mathfrak{g}$. Then there is a finitely generated commutative graded subalgebra $H^{\bullet}$ of $\mathrm{HH}^{*}(u(\mathfrak{g}, \chi))$ such that $H^{0}=\mathrm{HH}^{0}(u(\mathfrak{g}, \chi))$

and $\operatorname{Ext}_{u(\mathfrak{g}, \chi)}^{\cdot}(M, N)$ is finitely generated as an $H^{*}$-module for all finite dimensional $u(\mathfrak{g}, \chi)$-modules $M$ and $N$. 
Proof. According to [21, Theorem 2.4], there exists a natural isomorphism

$$
\operatorname{HH}^{\bullet}(u(\mathfrak{g}, \chi)) \cong \operatorname{Ext}_{u(\mathfrak{g}, \chi)^{e}}^{\cdot}(u(\mathfrak{g}, \chi), u(\mathfrak{g}, \chi)) \cong \operatorname{Ext}_{u(\mathfrak{g}, 0)}^{\cdot}(k, u(\mathfrak{g}, \chi)),
$$

where $u(\mathfrak{g}, \chi)$ is a restricted $\mathfrak{g}$-module (or equivalently, $u(\mathfrak{g}, \chi)$ is a $u(\mathfrak{g}, 0)$-module) via $x \cdot u:=x u-u x$ for every $x \in \mathfrak{g}$ and every $u \in u(\mathfrak{g}, \chi)$. It is clear that the unit map from $k$ to $u(\mathfrak{g}, \chi)$ is a homomorphism of restricted $\mathfrak{g}$-modules (i.e., a $u(\mathfrak{g}, 0)$ module homomorphism), and therefore there exists a natural homomorphism from $\operatorname{Ext}_{u(\mathfrak{g}, 0)}(k, k)$ into $\operatorname{Ext}_{u(\mathfrak{g}, 0)}(k, u(\mathfrak{g}, \chi)) \cong \mathrm{HH}^{*}(u(\mathfrak{g}, \chi))$. In particular, by virtue of $[\mathbf{2 6}$, Theorem 1.1], the factor algebra $E^{*}$ of $\operatorname{Ext}_{u(\mathfrak{g}, 0)}(k, k)$ modulo the kernel of this homomorphism is a finitely generated graded subalgebra of $\mathrm{HH}^{*}(u(\mathfrak{g}, \chi))$. Now set

$$
H^{\bullet}:= \begin{cases}\operatorname{HH}^{0}(u(\mathfrak{g}, \chi)) \cdot \bigoplus_{n=0}^{\infty} E^{n}, & \text { if } \operatorname{char} k=2, \\ \operatorname{HH}^{0}(u(\mathfrak{g}, \chi)) \cdot \bigoplus_{n=0}^{\infty} E^{2 n}, & \text { if } \operatorname{char} k \neq 2 .\end{cases}
$$

Then $H^{\bullet}$ is a finitely generated commutative graded subalgebra of $\mathrm{HH}^{*}(u(\mathfrak{g}, \chi))$ such that $H^{0}=\mathrm{HH}^{0}(u(\mathfrak{g}, \chi))$. Finally, the second part of the assertion follows from $[\mathbf{2 1}$, Corollary 2.5] and another application of [26, Theorem 1.1].

Theorem 2.1 and Corollary 2.2 in conjunction with [44, Corollary 5.4.3] and Lemma 4.1 imply [22, Theorem 4.1] and [22, Corollary 4.2], respectively. We give precise statements here for completeness.

Theorem 4.2. Let $\mathfrak{g}$ be a finite dimensional restricted Lie algebra over an algebraically closed field of prime characteristic, let $\chi$ be any linear form on $\mathfrak{g}$, and let $B$ be any block of the $\chi$-reduced universal enveloping algebra $u(\mathfrak{g}, \chi)$ of $\mathfrak{g}$. If there is a $B$-module $M$ such that $\mathrm{cx}_{u(\mathfrak{g}, \chi)}(M) \geqslant 3$, then $B$ is wild.

Corollary 4.3. Let $\mathfrak{g}$ be a finite dimensional restricted Lie algebra over an algebraically closed field of prime characteristic, let $\chi$ be any linear form on $\mathfrak{g}$, and let $B$ be any block of the $\chi$-reduced universal enveloping algebra $u(\mathfrak{g}, \chi)$ of $\mathfrak{g}$. If $B$ is tame, then $\operatorname{cx}_{u(\mathfrak{g}, \chi)}(M) \leqslant 2$ for every $B$-module $M$.

\section{Small half-quantum groups}

In this section, we give a wildness criterion for some small half-quantum groups, that is those corresponding to certain nilpotent subalgebras of complex simple Lie algebras. These are not Hopf algebras themselves, and so our main results in [23] do not apply directly.

Let $\mathfrak{g}$ be a finite dimensional complex simple Lie algebra, $\Phi$ its root system, and $r:=\operatorname{rk}(\Phi)$ its rank. Let $\ell>1$ be an odd integer and assume that $\ell$ is not divisible by 3 if $\Phi$ is of type $\mathrm{G}_{2}$. Let $q$ be a primitive complex $\ell$ th root of unity and let $u_{q}(\mathfrak{g})$ denote Lusztig's small quantum group [36].

Fix a set of simple roots and let $\Phi^{+}$and $\Phi^{-}$be the corresponding sets of positive and negative roots, respectively. Then $\mathfrak{g}$ has a standard Borel subalgebra corresponding to $\Phi^{+}$and an opposite standard Borel subalgebra corresponding to $\Phi^{-}$. Let $u_{q}^{\geqslant 0}(\mathfrak{g})$ (denoted $u_{q}^{+}(\mathfrak{g})$ in $[\mathbf{2 3}]$ ) be the Hopf subalgebra of $u_{q}(\mathfrak{g})$ corresponding to the standard Borel subalgebra of $\mathfrak{g}$ and let $u_{q}^{\leqslant 0}(\mathfrak{g})$ denote the Hopf subalgebra of 
$u_{q}(\mathfrak{g})$ corresponding to the opposite standard Borel subalgebra of $\mathfrak{g}$. Furthermore, we will use the notation $u_{q}^{>0}(\mathfrak{g})$ to denote the subalgebra of $u_{q}(\mathfrak{g})$ corresponding to the largest nilpotent ideal of the standard Borel subalgebra of $\mathfrak{g}$ and $u_{q}^{<0}(\mathfrak{g})$ to denote the subalgebra of $u_{q}(\mathfrak{g})$ corresponding to the largest nilpotent ideal of the opposite standard Borel subalgebra of $\mathfrak{g}$. Note that $u_{q}^{\leqslant 0}(\mathfrak{g}) \cong u_{q^{-1}}^{\geqslant 0}(\mathfrak{g})$ and $u_{q}^{<0}(\mathfrak{g}) \cong u_{q^{-1}}^{>0}(\mathfrak{g})$ (see $[\mathbf{1 3},(1.2 .10)])$.

Drupieski [18, Theorem 6.2.6] proved the following result for the full cohomology ring. In the usual way (see the argument before Theorem 3.1) one obtains from this the corresponding result for the even degree cohomology ring. In fact, Drupieski more generally deals with Frobenius-Lusztig kernels of quantum groups.

Theorem 5.1. Let $q$ be a primitive complex $\ell$ th root of unity and assume that $\ell>1$ is an odd integer not divisible by 3 if $\Phi$ is of type $\mathrm{G}_{2}$. Then the even cohomology ring $\mathrm{H}^{\mathrm{ev}}\left(u_{q}^{>0}(\mathfrak{g}), \mathbb{C}\right):=\bigoplus_{n \geqslant 0} \mathrm{H}^{2 n}\left(u_{q}^{>0}(\mathfrak{g}), \mathbb{C}\right)$ is finitely generated. Moreover, if $M$ is a finite dimensional $u_{q}^{>0}(\mathfrak{g})$-module, then $\mathrm{H}^{\bullet}\left(u_{q}^{>0}(\mathfrak{g}), M\right)$ is finitely generated as an $\mathrm{H}^{\mathrm{ev}}\left(u_{q}^{>0}(\mathfrak{g}), \mathbb{C}\right)$-module.

Let $G:=(\mathbb{Z} / \ell \mathbb{Z})^{r}$, a subgroup of the group of units of $u_{q}^{\geqslant 0}(\mathfrak{g})$. Then $u_{q}^{\geqslant 0}(\mathfrak{g})$ is a skew group algebra formed from its subalgebra $u^{>0}(\mathfrak{g})$ and the action of $G$ by conjugation. Consequently $G$ acts on the cohomology of $u_{q}^{>0}(\mathfrak{g})$. Let $H^{\bullet}:=\operatorname{HH}^{0}\left(u_{q}^{>0}(\mathfrak{g})\right)$. $\mathrm{H}^{\mathrm{ev}}\left(u_{q}^{>0}(\mathfrak{g}), \mathbb{C}\right)^{G}=\mathrm{HH}^{0}\left(u_{q}^{>0}(\mathfrak{g})\right) \cdot \operatorname{Ext}_{u_{q}^{>0}(\mathfrak{g})}^{\mathrm{ev}}(\mathbb{C}, \mathbb{C})^{G}$. By Theorem 5.1, $H^{\bullet}$ is finitely generated. We show next that it embeds into $\mathrm{HH}^{*}\left(u_{q}^{>0}(\mathfrak{g})\right)$; for details in a more general context, see the next section. By standard arguments, there is an isomorphism of Hochschild cohomology, $\mathrm{HH}^{*}\left(u_{q}^{\geqslant 0}(\mathfrak{g})\right) \cong \mathrm{HH}^{*}\left(u_{q}^{>0}(\mathfrak{g}), u_{q}^{\geqslant 0}(\mathfrak{g})\right)^{G}$. Similarly there is an isomorphism $\operatorname{Ext}_{u_{q} \geqslant 0(\mathfrak{g})}(\mathbb{C}, \mathbb{C}) \cong \operatorname{Ext}_{u_{q}^{\circ}(\mathfrak{g})}(\mathbb{C}, \mathbb{C})^{G}$. Since $u_{q}^{\geqslant 0}(\mathfrak{g})$ is a Hopf algebra, there is an embedding of $\operatorname{Ext}_{u_{q}^{\bullet}(\mathfrak{g})}(\mathbb{C}, \mathbb{C})$ into its Hochschild cohomology $\mathrm{HH}^{\bullet}\left(u_{q}^{\geqslant 0}(\mathfrak{g})\right)$ (see the last section for details and references). It can be checked that the image of $\operatorname{Ext}_{u_{q}^{>0}(\mathfrak{g})}^{\cdot}(\mathbb{C}, \mathbb{C})^{G}$ in $\mathrm{HH}^{*}\left(u_{q}^{>0}(\mathfrak{g}), u_{q}^{\geqslant 0}(\mathfrak{g})\right)^{G}$, under these isomorphisms, is contained in $\mathrm{HH}^{\bullet}\left(u_{q}^{>0}(\mathfrak{g})\right)^{G}$. Therefore (fg1) holds for $u_{q}^{>0}(\mathfrak{g})$. We now claim that by Theorem 5.1 and Remark 1.1, (fg2) holds for $u_{q}^{>0}(\mathfrak{g})$ : We must show that for all finite dimensional $u_{q}^{>0}(\mathfrak{g})$-modules $M$ and $N$, Ext ${ }_{u_{q}^{>0}(\mathfrak{g})}^{0^{\prime}}(M, N)$ is finitely generated as an $H^{\bullet}$-module. Since $N$ is a $u_{q}^{>0}(\mathfrak{g})$-direct summand of the module $u_{q}^{\geqslant 0}(\mathfrak{g}) \otimes_{u_{q}^{>0}(\mathfrak{g})} N$ induced to $u_{q}^{\geqslant 0}(\mathfrak{g})$ and restricted back to $u_{q}^{>0}(\mathfrak{g})$, this will be true if it is true for $N$ replaced by this induced module. By the Eckmann-Shapiro Lemma we have

$$
\operatorname{Ext}_{u_{q}^{>0}(\mathfrak{g})}\left(M, u_{q}^{\geqslant 0}(\mathfrak{g}) \otimes_{u_{q}^{>0}(\mathfrak{g})} N\right) \cong \operatorname{Ext}_{u_{q}^{\bullet}(\mathfrak{g})}\left(u_{q}^{\geqslant 0}(\mathfrak{g}) \otimes_{u_{q}^{>0}(\mathfrak{g})} M, u_{q}^{\geqslant 0}(\mathfrak{g}) \otimes_{u_{q}^{>0}(\mathfrak{g})} N\right) .
$$

The latter is finitely generated over $\operatorname{Ext}_{u_{q}^{\geqslant 0}(\mathfrak{g})}^{\mathrm{ev}}(\mathbb{C}, \mathbb{C}) \cong \operatorname{Ext}_{u_{q}^{>0}(\mathfrak{g})}^{\mathrm{ev}}(\mathbb{C}, \mathbb{C})^{G}$, which embeds into $H^{\bullet}$. Since the actions correspond under these isomorphisms, (fg2) does indeed hold for $u_{q}^{>0}(\mathfrak{g})$. For ease of reference we summarize this in the next result.

Corollary 5.2. Let $q$ be a primitive complex th root of unity and assume that $\ell>1$ is an odd integer not divisible by 3 if $\Phi$ is of type $\mathrm{G}_{2}$. Then the algebra $H^{*}:=$ $\mathrm{HH}^{0}\left(u_{q}^{>0}(\mathfrak{g})\right) \cdot \mathrm{H}^{\mathrm{ev}}\left(u_{q}^{>0}(\mathfrak{g}), \mathbb{C}\right)^{(\mathbb{Z} / \ell \mathbb{Z})^{r}}$ is finitely generated. Moreover, Ext. $\dot{u}_{u_{q}^{0}(\mathfrak{g})}(M, N)$ is finitely generated as an $H^{\bullet}$-module for all finite dimensional $u_{q}^{>0}(\mathfrak{g})$-modules $M$ and $N$. 
In order to be able to apply Theorem 2.1, we will need the following relation between the complexities of the trivial modules for $u_{q}^{>0}(\mathfrak{g})$ and for $u_{q}^{\geqslant 0}(\mathfrak{g})$.

Lemma 5.3. Let $q$ be a primitive complex $\ell$ th root of unity and assume that $\ell>1$ is an odd integer not divisible by 3 if $\Phi$ is of type $\mathrm{G}_{2}$. Then $\mathrm{cx}_{u_{q}^{>0}(\mathfrak{g})}(\mathbb{C})=\mathrm{cx}_{u_{q}^{>0}(\mathfrak{g})}(\mathbb{C})$.

Proof. Since $u_{q}^{\geqslant 0}(\mathfrak{g})$ is a free $u_{q}^{>0}(\mathfrak{g})$-module, every projective resolution of $u_{q}^{\geqslant 0}(\mathfrak{g})$ modules restricts to a projective resolution of $u_{q}^{>0}(\mathfrak{g})$-modules. Consequently, we have that $\mathrm{cx}_{u_{q}^{>0}(\mathfrak{g})}(\mathbb{C}) \leqslant \mathrm{cx}_{u_{q} \geqslant 0}(\mathfrak{g})(\mathbb{C})$.

On the other hand, it follows from $u_{q}^{\geqslant 0}(\mathfrak{g}) \cong(\mathbb{Z} / \ell \mathbb{Z})^{r} \cdot u_{q}^{>0}(\mathfrak{g})$, the Hochschild-Serre spectral sequence, and Maschke's Theorem that

$$
\mathrm{H}^{n}\left(u_{q}^{\geqslant 0}(\mathfrak{g}), \mathbb{C}\right) \cong \mathrm{H}^{n}\left(u_{q}^{>0}(\mathfrak{g}), \mathbb{C}\right)^{(\mathbb{Z} / \ell \mathbb{Z})^{r}}
$$

for every non-negative integer $n$. By Theorem $1.4, \operatorname{cx}_{A}(\mathbb{C})=\gamma\left(\operatorname{Ext}_{A}(\mathbb{C}, \mathbb{C})\right)$ for both $A=u_{q}^{\geqslant 0}(\mathfrak{g})$ and $A=u_{q}^{>0}(\mathfrak{g})$. We conclude that $\operatorname{cx}_{u_{q}^{>0}(\mathfrak{g})}(\mathbb{C}) \geqslant \operatorname{cx}_{u_{q}}(\mathfrak{g})(\mathbb{C})$, finishing the proof of the assertion.

The following theorem was proved by Cibils (see the proof of [10, Proposition 3.3]) in the simply laced case for any $\ell \geqslant 5$ by completely different methods.

Theorem 5.4. Let $r \geqslant 2$ and let $q$ be a primitive complex $\ell$ th root of unity. Assume that $\ell>1$ is an odd integer not divisible by 3 if $\Phi$ is of type $\mathrm{G}_{2}$. Then $u_{q}^{>0}(\mathfrak{g})$ is wild.

Note that the trivial module $\mathbb{C}$ is the only simple $u_{q}^{>0}(\mathfrak{g})$-module (up to isomorphism) and thus $u_{q}^{>0}(\mathfrak{g})$ has only one block. If $r=1$, then $u_{q}^{>0}(\mathfrak{g})$ is isomorphic to the truncated polynomial algebra $\mathbb{C}[X] /\left(X^{\ell}\right)$ and therefore $u_{q}^{>0}(\mathfrak{g})$ is representationfinite.

Proof. According to Lemma 5.3 and the proof of [23, Theorem 4.3], we obtain $\mathrm{Cx}_{u_{q}^{>0}(\mathfrak{g})}(\mathbb{C})=\mathrm{cx}_{u_{q}^{\geqslant 0}(\mathfrak{g})}(\mathbb{C}) \geqslant 3$ unless $r=1$. Since $u_{q}^{>0}(\mathfrak{g})$ is a Yetter-Drinfeld Hopf algebra over the group algebra $\mathbb{C}\left[(\mathbb{Z} / \ell \mathbb{Z})^{r}\right], u_{q}^{>0}(\mathfrak{g})$ is a Frobenius algebra (see $[\mathbf{2 4}$, Corollary 5.8] or [43, Proposition 2.10(3)]) and therefore self-injective. Thus, if $r \geqslant 2$, Theorem 2.1 in conjunction with Corollary 5.2 implies that $u_{q}^{>0}(\mathfrak{g})$ is wild.

Remark 5.5. A more direct proof for the fact that $u_{q}^{>0}(\mathfrak{g})$ is a Frobenius algebra can also be given along the lines of the proof of [9, Theorem 7.2] for the quantum Borel subalgebra $u_{q}^{\geqslant 0}(\mathfrak{g})$ by using the PBW-basis of the De Concini-Kac quantum group associated to $\mathfrak{g}[\mathbf{9}$, Section 6.1]. By this method one also finds that the Nakayama automorphism $u_{q}^{>0}(\mathfrak{g})$ is trivial.

Gordon [30, Theorem 7.1(b)(i)] proved the wildness of certain finite dimensional factor algebras of the quantized function algebra of a simply-connected connected complex semisimple algebraic group at roots of unity of odd degree (not divisible by 3 if the group has a component of type $\mathrm{G}_{2}$ ) by using Rickard's wildness criterion. We leave it to the interested reader to fix the gap in Gordon's proof by proceeding similarly to Section 4. Here [29, Theorem 5.2] plays the role of of [26, Theorem 1.1] in order to establish an analogue of Lemma 4.1 and [44, Corollary 5.4.3] is replaced by [9, Theorem 8.4]. Then Theorem 2.1 and [30, Theorem 6.20] show that the finite dimensional quantized function algebras in [30, Theorem 7.1(b)(i)] are wild. 
But note that in $[\mathbf{8}$, Theorem 4.5(ii)] the same result including the borderline case $l\left(w_{1}\right)+l\left(w_{2}\right)=2 N-2$ is proved by using completely different methods even without assuming that $\ell$ is larger than the Coxeter number.

\section{Nichols algebras}

In this section we apply Theorem 2.1 to Nichols algebras, also known as quantum symmetric algebras, generalizing the application to small half-quantum groups of the previous section. We summarize some of the definitions first; for more details, see, e.g., $[\mathbf{1}]$.

If $G$ is a finite group, then a Yetter-Drinfeld module over $k G$ is a $G$-graded vector space $V=\bigoplus_{g \in G} V_{g}$ that is also a $k G$-module for which $g \cdot V_{h}=V_{g h g^{-1}}$ for all $g, h \in$ $G$. The grading corresponds to a $k G$-comodule structure: $\delta(v)=g \otimes v$ for all $g \in G$ and $v \in V_{g}$. The category ${ }_{G}^{G} \mathcal{Y D}$ of all Yetter-Drinfeld modules over $k G$ is a braided monoidal category with braiding $c: U \otimes V \stackrel{\sim}{\rightarrow} V \otimes U$ determined by $c(u \otimes v)=g(v) \otimes$ $u$ whenever $g \in G, u \in U_{g}, v \in V$. A braided Hopf algebra in ${ }_{G}^{G} \mathcal{Y D}$ is an object $R$ of ${ }_{G}^{G} \mathcal{Y D}$ having structure maps (unit, multiplication, counit, comultiplication, coinverse) that are morphisms in the category and satisfy the usual commutative diagrams, e.g., the multiplication $\mu$ is associative in the sense that $\mu \circ\left(\mu \otimes \operatorname{id}_{R}\right)=\mu \circ\left(\operatorname{id}_{R} \otimes \mu\right)$. Examples of braided Hopf algebras in ${ }_{G}^{G} \mathcal{Y D}$ are the tensor algebra $T(V)$ and the Nichols algebra $\mathcal{B}(V)$ associated to a Yetter-Drinfeld module $V$ over $k G$ (the latter is defined to be a particular quotient of the tensor algebra $T(V)$ that can be finite dimensional).

Given a braided Hopf algebra $R$ in ${ }_{G}^{G} \mathcal{Y D}$, one may form its Radford biproduct (or its bosonization) $A:=R \# k G$ : As an algebra, this is just the skew group algebra, that is the free $R$-module with basis $G$ and multiplication $(r g)(s h)=r(g \cdot s) g h$ for $r, s \in R$ and $g, h \in G$. It is also a coalgebra, $\Delta(r g)=\sum r^{(1)}\left(r^{(2)}\right)_{(-1)} g \otimes\left(r^{(2)}\right)_{(0)} g$ for all $r \in R$ and $g \in G$, where $\Delta(r)=\sum r^{(1)} \otimes r^{(2)}$ in $R$ as a Hopf algebra in ${ }_{G}^{G} \mathcal{Y D}$ and $\delta(r)=\sum r_{(-1)} \otimes r_{(0)}$ is its $k G$-comodule structure. These two structures make $A$ into a Hopf algebra.

We assume that the characteristic of $k$ does not divide the order of $G$, and let $k$ be an $R$-module via the counit map from $R$ to $k$. We will be interested in the $G$-invariant subalgebra $\operatorname{Ext}_{R}{ }^{*}(k, k)^{G}$ of $\operatorname{Ext}_{R}(k, k)$. Some results in [38, Appendix] generalize to show that this subalgebra embeds into the Hochschild cohomology $\mathrm{HH}^{*}(R)$. We summarize these ideas here for completeness. We will use the fact that $\operatorname{Ext}_{A}^{\circ}(k, k) \cong \operatorname{Ext}_{R}^{\circ}(k, k)^{G}$, where $A=R \# k G$, valid since the characteristic of $k$ does not divide the order of $G$.

First define a map $\delta: A \rightarrow A^{e}$ by $\delta(a)=\sum a_{1} \otimes S\left(a_{2}\right)$ for every $a \in A$. This is an injective algebra homomorphism by [38, Lemma 11]. Let

$$
\mathfrak{D}:=\bigoplus_{g \in G}\left(R g \otimes R g^{-1}\right),
$$

a subalgebra of $A^{e}$. Note that since $R$ is in ${ }_{G}^{G} \mathcal{Y D}$, the algebra $\mathfrak{D}$ contains the subalgebra $\delta(A) \cong A$. The proof of [38, Lemma 11] shows that as induced modules from $\delta(A)$ to $\mathfrak{D}$ and to $A^{e}$, there are isomorphisms $\mathfrak{D} \otimes_{\delta(A)} k \cong R$ and $A^{e} \otimes_{\delta(A)} k \cong A$. Thus 
induction from $A \cong \delta(A)$ to $\mathfrak{D}$, and then to $A^{e}$, yields a sequence of isomorphisms

$$
\operatorname{Ext}_{A}^{\bullet}(k, k) \cong \operatorname{Ext}_{\mathfrak{D}}^{\bullet}(R, k) \cong \operatorname{Ext}_{A^{e}}(A, k) .
$$

The last space $\operatorname{Ext}_{A^{e}}(A, k)$ embeds, as an algebra, into $\operatorname{Ext}_{A^{e}}(A, A) \cong \mathrm{HH}^{\bullet}(A)$ via the unit map from $k$ to $A$ (see [28, Section 5.6] or [38, Lemma 12]). In fact, $\mathrm{HH}^{\circ}(A) \cong$ $\operatorname{Ext}_{R^{e}}(R, A)^{G} \cong \operatorname{Ext}_{\mathcal{D}}(R, A)$, and so the second of the above isomorphisms results in an embedding of $\operatorname{Ext}_{\mathfrak{D}}^{\circ}(R, k)$ into $\operatorname{Ext}_{\mathfrak{D}}(R, A)$. Its image is in $\operatorname{Ext}_{\mathfrak{D}}^{\bullet}(R, R) \cong \mathrm{HH}^{\bullet}(R)^{G}$, and the latter embeds into $\mathrm{HH}^{\circ}(R)$.

We will identify $\operatorname{Ext}_{R}(k, k)^{G}$ with its image in $\mathrm{HH}^{\bullet}(R)$ in what follows. Let

$$
H^{\bullet}:= \begin{cases}\operatorname{HH}^{0}(R) \cdot \bigoplus_{n=0}^{\infty} \operatorname{Ext}_{R}^{n}(k, k)^{G}, & \text { if char } k=2, \\ \operatorname{HH}^{0}(R) \cdot \bigoplus_{n=0}^{\infty} \operatorname{Ext}_{R}^{2 n}(k, k)^{G}, & \text { if char } k \neq 2,\end{cases}
$$

guaranteeing that $H^{\bullet}$ is a commutative algebra.

Theorem 6.1. Let $G$ be a finite group and let $k$ be an algebraically closed field whose characteristic does not divide the order of $G$. Let $R$ be a finite dimensional Hopf algebra in ${ }_{G}^{G} \mathcal{Y D}$. Assume (fg1) and (fg2) hold for $H^{\bullet}$ defined as above. If there is an $R$-module $M$ such that $\operatorname{cx}_{R}(M) \geqslant 3$, then $R$ is wild.

Proof. Since $A=R \# k G$ is a finite dimensional Hopf algebra, it is a Frobenius algebra. Standard arguments show that $R$ is itself a Frobenius algebra: $A$ is a free $R$-module, that is, $A \cong R^{n}$ as an $R$-module, where $n$ denotes the order of $G$. As $A$ is Frobenius, $A \cong A^{*}$ as $A$-modules, so $R^{n} \cong\left(R^{n}\right)^{*} \cong\left(R^{*}\right)^{n}$ as $R$-modules. By the Krull-Remak-Schmidt Theorem, $R \cong R^{*}$ as $R$-modules, and so $R$ is Frobenius. (For a proof in a much more general context, see [24, Corollary 5.8] or [43, Proposition $2.10(3)]$.) Since $R$ is Frobenius, it is self-injective, and the result now follows from Theorem 2.1 by choosing the block in which an indecomposable summand of $M$ of complexity at least 3 lies.

In order to apply Theorem 6.1, the following proposition may be useful in some cases. It is the analog of Lemma 5.3 in this setting.

Proposition 6.2. Let $G$ be a finite group and let $k$ be an algebraically closed field such that the characteristic of $k$ does not divide the order of $G$. Let $R$ be a finite dimensional Hopf algebra in ${ }_{G}^{G} \mathcal{Y D}$. Then $\mathrm{cx}_{R}(M)=\mathrm{cx}_{R \# k G}(M)$ for any $R \# k G$-module $M$.

Proof. The skew group algebra $R \# k G$ is free as an $R$-module, so a projective resolution of $M$ as an $R \# k G$-module restricts to a projective resolution of $M$ as an $R$-module. Therefore $\operatorname{cx}_{R}(M) \leqslant \operatorname{cx}_{R \# k G}(M)$. On the other hand, the $R \# k G$-module $M$ is a direct summand of the induced module $(R \# k G) \otimes_{R} M$ : Since the characteristic of $k$ does not divide the order $|G|$ of $G$, the canonical projection from this induced module to $M$ splits via the map $m \mapsto \frac{1}{|G|} \sum_{g \in G} g \otimes g^{-1} m$. Since a projective resolution of $M$ as an $R$-module may be induced to a projective resolution of $(R \# k G) \otimes_{R} M$ as an $R \# k G$-module, we now have

$$
\operatorname{cx}_{R}(M) \geqslant \operatorname{cx}_{R \# k G}\left((R \# k G) \otimes_{R} M\right) \geqslant \operatorname{cx}_{R \# k G}(M) .
$$


Alternatively, we may use the following lemma combined with results from [23], applied to the Hopf algebra $R \# k G$, to obtain a wildness criterion for Nichols algebras. The abelian case of the lemma is [8, Lemma 4.2]; the proof is valid more generally using Morita equivalence of $A$ and $(A \# k G) \#(k G)^{*}$ (see [11]).

Lemma 6.3. Let $G$ be a finite group and let $k$ be an algebraically closed field such that the characteristic of $k$ does not divide the order of $G$. Let $R$ be a finite dimensional algebra with an action of $G$ by automorphisms. Then $R$ and $R \# k G$ have the same representation type.

We next apply Theorem 6.1 to a large class of examples from [37]. These are the Nichols algebras and corresponding Hopf algebras arising in the classification of finite dimensional pointed Hopf algebras having abelian groups of group-like elements by Andruskiewitsch and Schneider [1]. Each such algebra is defined in terms of the following data: Let $\theta$ be a positive integer and let $\left(a_{i j}\right)_{1 \leqslant i, j \leqslant \theta}$ be a Cartan matrix of finite type, i.e., its Dynkin diagram is a disjoint union of copies of some of the diagrams $\mathrm{A}_{n}, \mathrm{~B}_{n}, \mathrm{C}_{n}, \mathrm{D}_{n}, \mathrm{E}_{6}, \mathrm{E}_{7}, \mathrm{E}_{8}, \mathrm{~F}_{4}, \mathrm{G}_{2}$. Let $\Phi$ be the root system corresponding to $\left(a_{i j}\right)$. Let $G$ be a finite abelian group and let $\widehat{G}$ be its dual group of characters. For each $i$, $1 \leqslant i \leqslant \theta$, choose $g_{i} \in G$ and $\chi_{i} \in \widehat{G}$ such that $\chi_{i}\left(g_{i}\right) \neq 1$ and $\chi_{j}\left(g_{i}\right) \chi_{i}\left(g_{j}\right)=\chi_{i}\left(g_{i}\right)^{a_{i j}}$ for all $1 \leqslant i, j \leqslant \theta$. Let $\mathcal{D}$ be the set of data $\left(G,\left(g_{i}\right)_{1 \leqslant i \leqslant \theta},\left(\chi_{i}\right)_{1 \leqslant i \leqslant \theta},\left(a_{i j}\right)_{1 \leqslant i, j \leqslant \theta}\right)$. The finite dimensional Hopf algebra $u(\mathcal{D})$ (also denoted $u(\mathcal{D}, 0,0)$ in [1]) is defined to be the Radford bosonization of the Nichols algebra $R$ corresponding to the following Yetter-Drinfeld module over $k G: V$ is a vector space with basis $x_{1}, \ldots, x_{\theta}$. For each $g \in G$, let $V_{g}$ be the $k$-linear span of all $x_{i}$ for which $g_{i}=g$, and let $g\left(x_{i}\right)=\chi_{i}(g) x_{i}$ for $1 \leqslant i \leqslant \theta$ and $g \in G$.

Example 6.4. Let $\mathfrak{g}$ be a complex simple Lie algebra of rank $\theta$, let $\left(a_{i j}\right)$ be the corresponding Cartan matrix, and let $\alpha_{1}, \ldots, \alpha_{\theta}$ be distinct simple roots. Let $q$ be a primitive $\ell$ th root of unity, $\ell$ odd and prime to 3 if $\mathfrak{g}$ has a component of type $\mathrm{G}_{2}$. Let $G:=(\mathbb{Z} / \ell \mathbb{Z})^{\theta}$ with generators $g_{1}, \ldots, g_{\theta}$. Let $(-,-)$ denote the positive definite symmetric bilinear form on the real vector space spanned by the simple roots $\alpha_{1}, \ldots, \alpha_{\theta}$ that is induced from the Killing form of $\mathfrak{g}$. For each $i$, set $\chi_{i}\left(g_{j}\right):=q^{\left(\alpha_{i}, \alpha_{j}\right)}$ and extend this to a character on $G$. The Nichols algebra $R$ is $u_{q}^{>0}(\mathfrak{g})$ and its Radford bosonization is $R \# k G \cong u_{q}^{\geqslant 0}(\mathfrak{g})$.

We assume that the order $N_{i}$ of $\chi_{i}\left(g_{i}\right)$ is odd for all $i$, and is prime to 3 for all $i$ in a connected component of type $\mathrm{G}_{2}$.

Theorem 6.5. Let $R$ be the Nichols algebra and let $u(\mathcal{D})$ be the complex Hopf algebra described above in terms of the data $\mathcal{D}$ with $\theta \geqslant 2$. Assume that $\chi_{i}^{N_{i}}$ is the trivial character for all $i$ and that the only common solution $\left(c_{1}, \ldots, c_{\theta}\right) \in\{0,1\}^{\theta}$ of the equations

$$
\chi_{1}(g)^{c_{1}} \cdots \chi_{\theta}(g)^{c_{\theta}}=1
$$

for all $g \in G$ is $\left(c_{1}, \ldots, c_{\theta}\right)=(0, \ldots, 0)$. Then $R$ and $u(\mathcal{D})$ are both wild.

We note that under some conditions, $u_{q}^{>0}(\mathfrak{g})$ and $u_{q}^{\geqslant 0}(\mathfrak{g})$ are included among the Nichols algebras and Hopf algebras of the form $R$ and $u(\mathcal{D})$ satisfying the hypotheses of the theorem: For these classes of examples, $\chi_{i}\left(g_{i}\right)=q^{\left(\alpha_{i}, \alpha_{i}\right)}$, whose order $N_{i}$ is 
just the order $\ell$ of $q$. Thus $\chi_{i}^{N_{i}}$ is indeed the trivial character for all $i$. A common solution $\left(c_{1}, \ldots, c_{\theta}\right)$ of the equations $\chi_{1}(g)^{c_{1}} \cdots \chi_{\theta}(g)^{c_{\theta}}=1$ for all $g \in G$ is equivalent to a common solution for the generators $g=g_{j}$ for all $j$, which is a solution to the equations

$$
\left(\alpha_{1}, \alpha_{j}\right) c_{1}+\cdots+\left(\alpha_{\theta}, \alpha_{j}\right) c_{\theta} \equiv 0 \quad(\bmod \ell)
$$

for all $j$. Ginzburg and Kumar [28, Section 2.5, pp. 187-188] showed that under their hypotheses, no non-zero solution exists in $\{0,1\}^{\theta}$ : They assume that the order $\ell$ of the root of unity $q$ is odd, greater than the Coxeter number of $\mathfrak{g}$, and prime to 3 if $\mathfrak{g}$ has a component of type $\mathrm{G}_{2}$. This provides an alternative approach to the results in Section 5 .

Proof of Theorem 6.5. Let

$$
H^{\bullet}:= \begin{cases}\operatorname{HH}^{0}(R) \cdot \bigoplus_{n=0}^{\infty} \operatorname{Ext}_{u(\mathcal{D})}^{n}(k, k), & \text { if } \operatorname{char} k=2, \\ \operatorname{HH}^{0}(R) \cdot \bigoplus_{n=0}^{\infty} \operatorname{Ext}_{u(\mathcal{D})}^{2 n}(k, k), & \text { if } \operatorname{char} k \neq 2 .\end{cases}
$$

By [37, Corollary 5.5], Ext ${ }_{u(\mathcal{D})}^{\cdot}(k, k)$ is isomorphic to the $G$-invariant subalgebra of a polynomial algebra in $\left|\Phi^{+}\right| \geqslant 3$ indeterminates (in cohomological degree 2), and the group $G$ acts diagonally on these indeterminates. Therefore assumption (fg1) holds for $R$. Similarly, for $u(\mathcal{D})$, we take $H^{\bullet}$ as for $R$ but replace the factor $\operatorname{HH}^{0}(R)$ with $\mathrm{HH}^{0}(u(\mathcal{D}))$, and see that (fg1) holds for $u(\mathcal{D})$.

We claim that by Remark 1.1, (fg2) holds for $R$ : By [37, Theorem 5.3], (fg2) holds for the corresponding Hopf algebra $u(\mathcal{D}) \cong R \# k G$, since

$$
\operatorname{Ext}_{u(\mathcal{D})}^{\cdot}(U, V) \cong \operatorname{Ext}_{u(\mathcal{D})}^{\bullet}\left(k, V \otimes U^{*}\right)
$$

for all finite dimensional $u(\mathcal{D})$-modules $U$ and $V$. Next, let $N$ be a finite dimensional $R$-module. By the Eckmann-Shapiro Lemma,

$$
\operatorname{Ext}_{R \# k G}^{\cdot}\left((R \# k G) \otimes_{R} M,(R \# k G) \otimes_{R} N\right) \cong \operatorname{Ext}_{R}^{\cdot}\left(M,(R \# k G) \otimes_{R} N\right) .
$$

Now $N$ is a direct summand of $(R \# k G) \otimes_{R} N$ as an $R$-module; therefore, $\operatorname{Ext}_{R}(M, N)$ is finitely generated over $\operatorname{Ext}_{R \# k G}^{\circ}(k, k) \cong \operatorname{Ext}_{R}^{\cdot}(k, k)^{G}$ since $\operatorname{Ext}_{R}\left(M,(R \# k G) \otimes_{R}\right.$ $N)$ is. Under our assumptions, $\operatorname{cx}_{R}(k) \geqslant 3$, so by Theorem $6.1, R$ is wild. Replacing $R$ by $u(\mathcal{D})$, we obtain the analogous conclusion for $u(\mathcal{D})$.

The hypothesis regarding solutions $\left(c_{1}, \ldots, c_{\theta}\right)$ in the theorem is surely not necessary. However, in general, it is more difficult to determine precisely the Krull dimension of the relevant $G$-invariant cohomology ring.

\section{Comparison of varieties for Hopf algebras}

In this section, we look at finite dimensional Hopf algebras in general, explaining the connection between the varieties defined in terms of Hochschild cohomology used in this article and those defined in terms of the cohomology of the trivial module used in $[\mathbf{2 3}]$.

Let $A$ be a finite dimensional Hopf algebra over $k$. Then $A$ is a Frobenius algebra and therefore self-injective. Let $\mathrm{H}^{\circ}(A, k):=\operatorname{Ext}_{A}(k, k)$, and let 


$$
\mathrm{H}^{\mathrm{ev}}(A, k):= \begin{cases}\bigoplus_{n=0}^{\infty} \mathrm{H}^{n}(A, k), & \text { if } \operatorname{char} k=2, \\ \bigoplus_{n=0}^{\infty} \mathrm{H}^{2 n}(A, k), & \text { if } \operatorname{char} k \neq 2 .\end{cases}
$$

We will need the following assumption, as in [23].

Assumption (fg). Assume that $\mathrm{H}^{\mathrm{ev}}(A, k)$ is finitely generated, and that, for any two finite dimensional $A$-modules $M$ and $N$, the $\mathrm{H}^{\mathrm{ev}}(A, k)$-module $\operatorname{Ext}_{A}(M, N)$ is finitely generated.

In [23], the cohomology ring $\mathrm{H}^{\mathrm{ev}}(A, k)$ was used to define varieties for modules: For each $A$-module $M, \mathcal{V}_{A}(M)$ is the maximal ideal spectrum of $\mathrm{H}^{\mathrm{ev}}(A, k) / I_{A}(M)$, where $I_{A}(M)$ is the annihilator of the action of $\mathrm{H}^{\mathrm{ev}}(A, k)$ on $\operatorname{Ext}_{A}(M, M)$.

As mentioned in Section 6, the cohomology ring $\mathrm{H}^{\circ}(A, k)$ embeds into Hochschild cohomology $\mathrm{HH}^{*}(A)$ as a graded subalgebra (see [28, Section 5.6] or [38, Lemma 12]). Let $B_{0}$ be the principal block of $A$. Note that $\mathrm{H}^{*}(A, k)$ in fact embeds into $\mathrm{HH}^{*}\left(B_{0}\right)$. Under assumption (fg), let

$$
H^{\bullet}:=\operatorname{HH}^{0}\left(B_{0}\right) \cdot \mathrm{H}^{\mathrm{ev}}(A, k),
$$

that is, $H^{\bullet}$ is the subalgebra of $\mathrm{HH}^{*}\left(B_{0}\right)$ generated by $\mathrm{HH}^{0}\left(B_{0}\right)$ and the image of $\mathrm{H}^{\mathrm{ev}}(A, k)$ in $\mathrm{HH}^{*}\left(B_{0}\right)$. Then $H^{*}$ satisfies (fg1) and (fg2). By Theorem 1.4, Lemma 1.3, and [23, Proposition 2.3], for any finite dimensional $B_{0}$-module $M$,

$$
\operatorname{dim} \mathcal{V}_{H}(M)=\operatorname{cx}_{B_{0}}(M)=\operatorname{cx}_{A}(M)=\operatorname{dim} \mathcal{V}_{A}(M) .
$$

That is, $\operatorname{dim} \mathcal{V}_{H}(M)=\operatorname{dim} \mathcal{V}_{A}(M)$, as one expects, since $\mathrm{HH}^{0}\left(B_{0}\right) \cong Z\left(B_{0}\right)$ is finite dimensional. In fact, the varieties $\mathcal{V}_{H}(M)$ and $\mathcal{V}_{A}(M)$ are isomorphic. We explain this next.

By [38, Lemma 13], the following diagram commutes for every finite dimensional $A$-module $M$ :

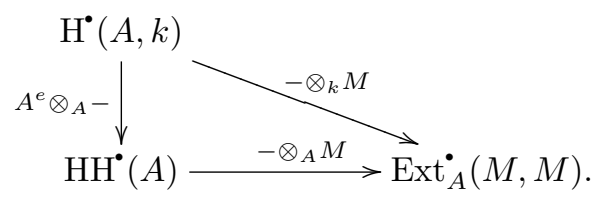

The vertical arrow is an embedding that is induced by an embedding from $A$ to $A^{e}$ (see, e.g., [38, Lemma 11 and Lemma 12]). This diagram shows that the action of $\mathrm{H}^{\mathrm{ev}}(A, k)$ on $\operatorname{Ext}_{A}(M, M)$ factors through $\mathrm{HH}^{*}(A)$, resulting in a commutative diagram

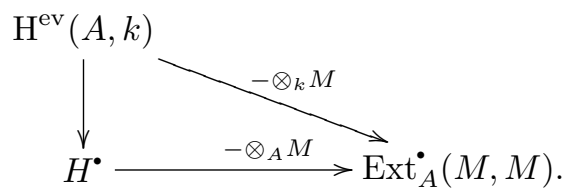

Again, the vertical arrow is an embedding and induces an embedding

$$
\mathrm{H}^{\mathrm{ev}}(A, k) / I_{A}(M) \longrightarrow H^{\bullet} / I_{H}(M) \text {. }
$$

As $\operatorname{HH}^{0}\left(B_{0}\right) \cong Z\left(B_{0}\right)$ is a local algebra, the above embedding induces an isomorphism modulo radicals. Thus $\mathcal{V}_{H}(M)$ and $\mathcal{V}_{A}(M)$ are isomorphic. 
Applying Theorem 2.1 and Corollary 2.2, in light of (1), we recover versions of [ 23 , Theorem 3.1 and Corollary 3.2] for the principal block. These results were originally proven using Hopf-theoretic techniques. In particular, tensor products of modules were used in [23, Corollary 2.6], crucial in the proofs of [23, Theorem 3.1 and Corollary 3.2].

Remark 7.1. In case $G$ is a finite group and $A=k G$, much more is true: Linckelmann [33] proved that one may take $H^{*}$ instead to be the full even Hochschild cohomology ring $\mathrm{HH}^{\mathrm{ev}}\left(B_{0}\right)$ and still obtain a isomorphism between the varieties $\mathcal{V}_{H}(M)$ and $\mathcal{V}_{A}(M)$. More generally, Linckelmann defined block cohomology for non-principal blocks in this setting and proved that the resulting variety of a module is isomorphic to that obtained via Hochschild cohomology of the block.

\section{References}

[1] N. Andruskiewitsch and H.-J. Schneider, On the classification of finitedimensional pointed Hopf algebras, Ann. of Math. (2) 171 (2010), no. 1, 375417.

[2] S. Ariki, Hecke algebras of classical type and their representation type, Proc. London Math. Soc. (3) 91 (2005), no. 2, 355-413; Corrigendum, Proc. London Math. Soc. (3) 92 (2006), no. 2, 342-344.

[3] S. Ariki and A. Mathas, The representation type of Hecke algebras of type $B$, Adv. Math. 181 (2004), no. 1, 134-159; Corrigendum, Adv. Math. 192 (2005), no. $1,228-230$.

[4] M. Auslander, I. Reiten, and S.O. Smalø, Representation theory of Artin algebras (Corrected reprint of the 1995 original), Cambridge Studies in Advanced Mathematics, vol. 36, Cambridge University Press, Cambridge, 1997.

[5] D.J. Benson, Representations and Cohomology I: Basic Representation Theory of Finite Groups and Associative Algebras, Cambridge Studies in Advanced Mathematics, vol. 30, Cambridge University Press, Cambridge, 1991.

[6] D.J. Benson, K. Erdmann, and A. Mikaelian, Cohomology of Hecke algebras, Homology Homotopy Appl. 12 (2010), no. 2, 353-370.

[7] P.A. Bergh and Ø. Solberg, Relative support varieties, Q. J. Math. 61 (2010), no. 2, 171-182.

[8] K.A. Brown and I.G. Gordon, The ramifications of the centres: quantised function algebras at roots of unity, Proc. London Math. Soc. (3) 84 (2002), no. 1, $147-178$.

[9] K.A. Brown, I.G. Gordon, and C.H. Stroppel, Cherednik, Hecke and quantum algebras as free Frobenius and Calabi-Yau extensions, J. Algebra 319 (2008), no. 3, 1007-1034.

[10] C. Cibils, Half-quantum groups at roots of unity, path algebras, and representation type, Internat. Math. Res. Notices 1997, no. 12, 541-553.

[11] M. Cohen and S. Montgomery, Group-graded rings, smash products, and group actions, Trans. Amer. Math. Soc. 282 (1984), no. 1, 237-258. 
[12] W. Crawley-Boevey, On tame algebras and bocses, Proc. London Math. Soc. (3) 56 (1988), no. 3, 451-483.

[13] C. De Concini and V.G. Kac, Representations of quantum groups at roots of 1, in Operator Algebras, Unitary Representations, Enveloping Algebras, and Invariant Theory (Paris, 1989) (eds. A. Connes, M. Duflo, A. Joseph, and R. Rentschler), Progr. Math., vol. 92, Birkhäuser, Boston, MA, 1990, pp. 471-506.

[14] R. Dipper and J. Du, Trivial and alternating source modules of Hecke algebras of type A, Proc. London Math. Soc. (3) 66 (1993), no. 3, 479-506.

[15] R. Dipper and G. James, Representations of Hecke algebras of general linear groups, Proc. London Math. Soc. (3) 52 (1986), no. 1, 20-52.

[16] R. Dipper and G. James, Blocks and idempotents of Hecke algebras of general linear groups, Proc. London Math. Soc. (3) 54 (1987), no. 1, 57-82.

[17] R Dipper and G. James, Representations of Hecke algebras of type $\mathrm{B}_{n}, J$. Algebra 146 (1992), no. 2, 454-481.

[18] C.M. Drupieski, Representations and cohomology for Frobenius-Lusztig kernels, J. Pure Appl. Algebra 215 (2011), no. 6, 1473-1491.

[19] K. Erdmann, M. Holloway, N. Snashall, Ø. Solberg, and R. Taillefer, Support varieties for selfinjective algebras, K-Theory 33 (2004), no. 1, 67-87.

[20] K. Erdmann and D.K. Nakano, Representation type of Hecke algebras of type A, Trans. Amer. Math. Soc. 354 (2002), no. 1, 275-285.

[21] R. Farnsteiner, Cohomology groups of reduced enveloping algebras, Math. Z. 206 (1991), no. 1, 103-117.

[22] R. Farnsteiner, Tameness and complexity of finite group schemes, Bull. London Math. Soc. 39 (2007), no. 1, 63-70.

[23] J. Feldvoss and S. Witherspoon, Support varieties and representation type of small quantum groups, Internat. Math. Res. Notices 2010, no. 7, 1346-1362.

[24] D. Fischman, S.M. Montgomery, and H.-J. Schneider, Frobenius extensions of subalgebras of Hopf algebras, Trans. Amer. Math. Soc. 349 (1997), no. 12, $4857-4895$.

[25] E.M. Friedlander and J. Pevtsova, Representation-theoretic support spaces for finite group schemes, Amer. J. Math. 127 (2005), no. 2, 379-420; Erratum, Amer. J. Math. 128 (2006), no. 4, 1067-1068.

[26] E.M. Friedlander and A. Suslin, Cohomology of finite group schemes over a field, Invent. Math. 127 (1997), no. 2, 209-270

[27] M. Gerstenhaber, The cohomology structure of an associative ring, Ann. of Math. (2) 78 (1963), no. 2, 267-288.

[28] V. Ginzburg and S. Kumar, Cohomology of quantum groups at roots of unity, Duke Math. J. 69 (1993), no. 1, 179-198.

[29] I.G. Gordon, Cohomology of quantized function algebras at roots of unity, Proc. London Math. Soc. (3) 80 (2000), no. 2, 337-359.

[30] I.G. Gordon, Complexity of representations of quantised function algebras and representation type, J. Algebra 233 (2000), no. 2, 437-482. 
[31] I.G. Gordon and A. Premet, Block representation type of reduced enveloping algebras, Trans. Amer. Math. Soc. 354 (2002), no. 4, 1549-1581.

[32] J. Hu, A Morita equivalence theorem for Hecke algebra $\mathcal{H}_{q}\left(\mathrm{D}_{n}\right)$ when $n$ is even, Manuscripta Math. 108 (2002), no. 4, 409-430.

[33] M. Linckelmann, Hochschild and block cohomology varieties are isomorphic, $J$. Lond. Math. Soc. (2) 81 (2010), no. 2, 389-411.

[34] M. Linckelmann, Finite generation of Hochschild cohomology of Hecke algebras of finite classical type in characteristic zero, Bull. London Math. Soc. 43 (2011), 871-885.

[35] J.-L. Loday, Cyclic Homology (2nd edition), Grundlehren der mathematischen Wissenschaften, vol. 301, Springer-Verlag, New York, 1998.

[36] G. Lusztig, Finite-dimensional Hopf algebras arising from quantized universal enveloping algebras, J. Amer. Math. Soc. 3 (1990), no. 1, 257-296.

[37] M. Mastnak, J. Pevtsova, P. Schauenburg, and S. Witherspoon, Cohomology of finite-dimensional pointed Hopf algebras, Proc. London Math. Soc. (3) 100 (2010), no. 2, 377-404.

[38] J. Pevtsova and S. Witherspoon, Varieties for modules of quantum elementary abelian groups, Algebr. Represent. Theory 12 (2009), no. 6, 567-595.

[39] C. Pallikaros, Representations of Hecke algebras of type $\mathrm{D}_{n}$, J. Algebra 169 (1994), no. 1, 20-48.

[40] A. Premet, Complexity of Lie algebra representations and nilpotent elements of the stabilizers of linear forms, Math. Z. 228 (1998), no. 2, 255-282.

[41] J. Rickard, The representation type of self-injective algebras, Bull. London Math. Soc. 22 (1990), no. 6, 540-546.

[42] N. Snashall and Ø. Solberg, Support varieties and Hochschild cohomology rings, Proc. London Math. Soc. (3) 88 (2004), no. 3, 705-732.

[43] Y. Sommerhäuser, Ribbon transformations, integrals, and triangular decompositions, J. Algebra 282 (2004), no. 2, 423-489.

[44] H. Strade and R. Farnsteiner, Modular Lie algebras and their representations, Monographs and Textbooks in Pure and Applied Mathematics, vol. 116, Marcel Dekker, Inc., New York, 1988.

[45] A. Suslin, E.M. Friedlander, and C.P. Bendel, Support varieties for infinitesimal group schemes, J. Amer. Math. Soc. 10 (1997), no. 3, 729-759.

Jörg Feldvoss jfeldvoss@jaguar1.usouthal.edu

Department of Mathematics and Statistics, University of South Alabama, Mobile, AL 36688-0002.

Sarah Witherspoon sjw@math.tamu.edu

Department of Mathematics, Texas A\&M University, College Station, TX 778433368 . 